\title{
Experimental Study of the Earthquake Recurrence Period and the Trend of Post-seismic Development
}

\author{
Bin Wang, ${ }^{1,2}$ Zhaoyong Xu, ${ }^{2}$ Xiangchu Yin, ${ }^{3}$ Yili Hu, ${ }^{4}$ Runhai Yang, ${ }^{2}$ \\ JingGuAN CAI, ${ }^{2}$ and SHUNYUn $\mathrm{CHEN}^{2}$
}

\begin{abstract}
In order to study the earthquake recurrence and the characteristics of earthquake series, rupture tests of rock samples and plexiglass samples were made. On rock samples, a number of acoustic emission (AE) and strain measuring points were deployed; the load was one side direct shear. The variation characteristics of AE and strain at different detecting points around the extra large fracture were observed and studied. On plexiglass samples, a series of inclined cracks were prefabricated by a small-scale compressive testing machine. The samples were then loaded on a shockproof platen, when the samples were loaded, the stress intensity factor (SIF) was determined by the laser interferometric technique and shadow optical method of caustics. The fracture conditions such as material toughness around the extra large fracture were also studied. From those experimental results and the theory of fracture mechanics, the earthquake recurrence period and the trend of post-seismic development were studied.
\end{abstract}

Key words: Earthquake period, post-seismic trend, experimental study, stress intensity factor, strain energy, low-frequency wave.

\section{Introduction}

After an earthquake has occurred, will still greater shocks occur? This problem is very important, both for the victims and for the government. Many experts have studied the types of earthquakes' series. The most basic types are the foremain-aftershock type, fore-mainshock type, main-aftershock type and swarm type. They have also proposed methods to judge the earthquake type. For example, the well-known Mogi's experiments (MoGI, 1962, 1963) simulated the foreshockmainshock-aftershock type by the fracture of homogeneous materials. Although

\footnotetext{
${ }^{1}$ School of Earth and Space Sciences, University of Science and Technology of China, Hefei 230026, PRC. E-mail: wbin8@163.com

${ }^{2}$ Disaster Prevention Research Institute of Yunnan Province, Kunming 650041, PRC. E-mail: zhaoyongx@126.com; renhaiyang@163.com

${ }^{3}$ Institute of Mechanics, Chinese Academy of Science, LNM, Beijing 100080, PRC. E-mail: xcyinlurr@vip.163.com; xcyin@public.bta.net.cn

${ }^{4}$ Department of Geophysics, Yunnan University, Kunming 650091, PRC. E-mail: ylhu929@163.com
} 
there are some drawbacks in his experiments examined from the present point of view, he has set a precedent for studying the types of earthquakes by experiments. The principle used for seismic zonation is that earthquakes are likely to occur repeatedly at the same place or in its vicinity. In earthquake prediction, the concept of long-term (such as 20 50 a) alternation of earthquake active and quiet periods is frequently used. There are even some results of special studies of the recurrence period of earthquakes (Mei Shirong, 1960; Shi Zhenliang et al., 1984; XU Shaoxie, 1982; Zhang Guomin, 1987; Zhang Guomin et al., 1993; Cai Jingguan and LiU ZHENGRONG, 1990). However, being limited by the conditions, experimental and theoretical confirmation of these results has not been done fully. In view of this, we improved the experimental conditions and designed some new experiments. First, rupture tests of rock samples, on which a number of $\mathrm{AE}$ and strain measuring points were deployed, were made by one side direct shear; the purpose was to induce ruptures of the samples producing various types of earthquakes. Second, plexiglass samples that contain a number of prefabricated inclined cracks were loaded by a small-scale compressive testing machine which was placed on a shockproof platen; the SIFs were determined by the laser interferometric technique and shadow optical method of caustics and the signals of microcracking were detected. At the same time, some indicative physical quantities in those experiments were recorded. These results were taken as an experimental basis, consequently a theoretical study was made.

\section{Experimental Methods}

\subsection{One Side Direct Shear Test of Rock Samples}

Direct shear tests were made on rectangular parallelepiped rock samples. The sample materials were sandstone and granite, which were pieced together into rectangular parallelepiped $80 \sim 120 \mathrm{~mm}$ by $40 \sim 60 \mathrm{~mm}$ by $60 \sim 80 \mathrm{~mm}$ in size. The strength of sandstone is lower and that of granite is higher. On the free side surfaces of the sample, 12 strain measuring points and $8 \mathrm{AE}$ measuring points were deployed (see Fig. 1b). The sample was placed on a true triaxial compressive testing machine, its lower half was clamped firmly from the two free side surfaces. In the mid-height, a gap of $1 \sim 3 \mathrm{~mm}$ wide was arranged in advance for shear fracture. A push was applied on one of the free side surfaces of the upper half of the sample, with the opposite surface left free. On the top surface, there was a combination of two steel blocks, with 2 4 (depending on the size of sample) rollers in between; a pressure transducer was placed above it. A normal stress of about $4 \sim 8 \mathrm{MPa}$ in the vertical direction was applied first. The normal stress was kept stable after it had reached the prescribed value, and a push was applied in the horizontal direction to do the direct shear test up to fracture (see Fig. 1a). In the beginning, the loading rate was a bit slower, about $4 \mathrm{MPa} / \mathrm{min}$. After the shear stress had reached about 

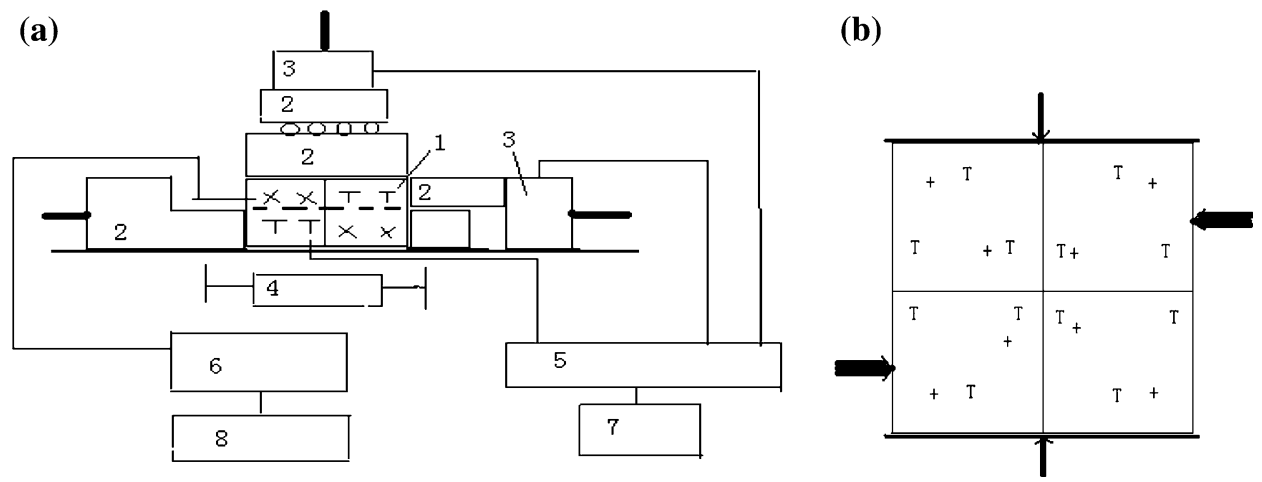

Figure 1

A block diagram showing the setup of direct shear test up to rupture (a), and a sketch of the distribution of measuring points (b). (a) 1. Rock sample (sandstone and granite pieced together); 2. Steel block; 3. Pressure transducer; 4. Displacement transducer; 5. Strainmeter; 6. Transient wave memory; 7, 8. Computer. (b) T: strain gage; + AE transducer; Vertical arrow: normal pressure; Horizontal thick arrow: shear load.

$50 \%$ of the shear strength, the loading rate was raised. The strain gages were parallel to the horizontal push and were connected to the Wheatstone bridge circuit as the active gage in the half bridge. The LB-2 digital strainmeter designed by the Institute of Geology of China Seismological Administration was used to record the pressure, displacement and strain. The LB-2 digital strainmeter is capable of continuous sampling; it has 16 channels and each channel has a sampling rate that can be adjusted between $1 \sim 1,000 \mathrm{~Hz}$ and a resolution of 16 bit. In the meantime, the AE information was recorded synchronously by the microcrack-recording system that consists of detector, amplifier, transient wave memory, and so on (XU et al., 2002a,b).

\subsection{Laser Interferometric Experiment of Plexiglass Samples}

The sample was made of plexiglass and was $200 \mathrm{~mm}$ by $200 \mathrm{~mm}$ by $15 \mathrm{~mm}$ in size. On the sample, a number of inclined penetrating cracks were cut by laser. The cracks make an angle of about $30^{\circ}$ with the direction of the major principal stress $\sigma_{1}$ $\left(\sigma_{y}\right)$ and are $35 \mathrm{~mm}$ long and $0.1 \sim 0.3 \mathrm{~mm}$ wide. The cracks (faults) were arranged in an en echelon array to form $Y$-shaped block boundaries. A small-scale compressive testing machine, capable of applying vertical and horizontal pressures respectively up to $300 \mathrm{KN}$, was installed on a shockproof platen. At the beginning of the experiment, horizontal and vertical pressures (in $x$ and $y$ directions, respectively) were synchronously applied to the sample. When the pressures had reached a certain degree (letting $\sigma_{x}=\sigma_{y}=5 \mathrm{MPa}$ ), $\sigma_{x}$ was kept approximately unchanged. Then, $\sigma_{y}$ was increased continuously until the final rupture of the sample. In another case, the 
(a)

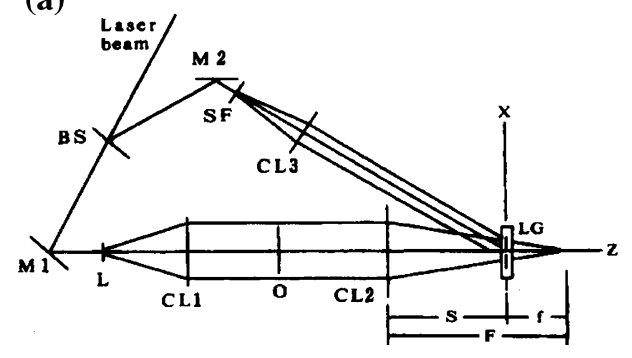

(b)

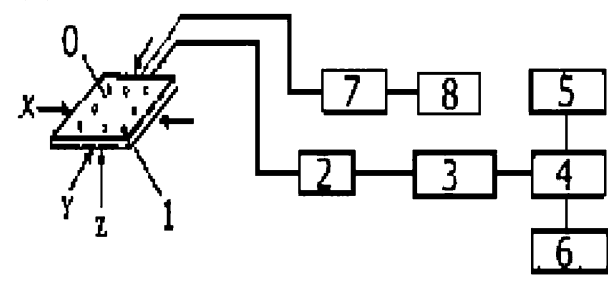

Figure 2

(a) Experimental setup of photography by real-time holographic interferometry BS: beam splitter; M1, M2: mirror; SF: spatial filter; L, CL1, CL2, CL3: lens; LG: liquid gate; O: transparent plexiglass sample. (b) Diagram showing the method of loading and the arrangement of transducers 0 Prefabricated crack; 1 PZT transducer; 2 amplifier; 3 transient wave memory; 4 and 8 computer; 5 printer; 6 plotter; 7 strainmeter.

sample was unloaded when $\sigma_{y}$ had been increased to about $70 \%$ of the rupture stress. From the beginning of loading, the strain patterns were recorded photographically by the real-time holographic interferometry (Fig. 2a) (XIONG et al., 2002); at the same time, the AE activity was recorded by the system for microcracking information storage and analysis (Fig. 2b) (Xu et al., 2002a,b).

\section{Test Results}

\subsection{Characteristics of Stress-strain Variations in the Rock Sample During the Loading Process}

Figure 3 a shows the variations of stress and strain with time in the rock sample during the loading process. Among the curves, one shows the horizontal shear stress (the dark line in Fig. 3), and those remaining show the strains of measuring points on the rock sample. Figure $3 \mathrm{~b}$ gives the stress-strain curve of horizontal shear. In Figure 3, except the tendentious variations, there are three large and obvious sudden changes, among which the latter two are even more remarkable; they respectively correspond to extra large fractures. When viewed from the figure, the general trend is that the shear force and strains at various points increase gradually with time. The modes of strain variation around the three extra fractures are not the same. After the first fracture, the strains of many points increased drastically; in contrast, after the latter two fractures, the strains of points all decreased drastically. In addition, when the three especially clear extra large fractures were impending, the various strain measuring points showed characteristic variations: the strain of some points increased or decreased following the original trend; the strain of other points changed the trend, from increasing to decreasing or the opposite; whereas there are 


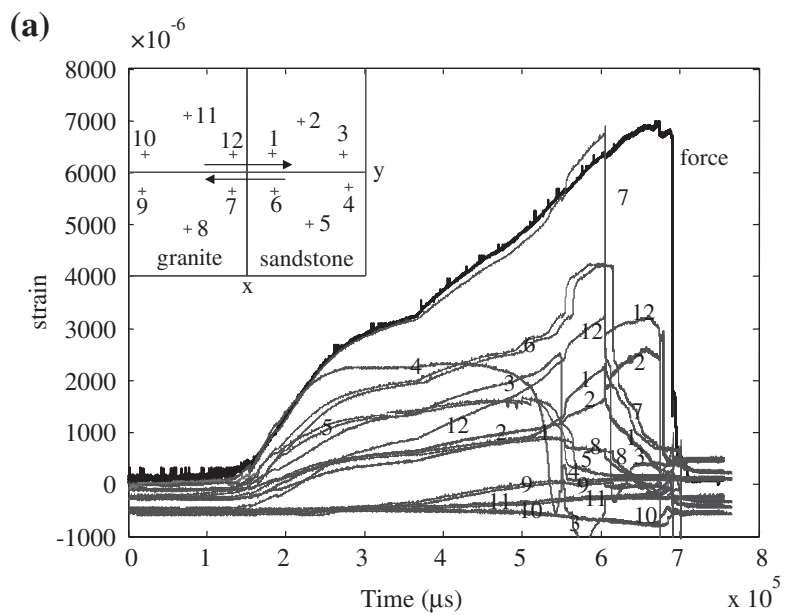

(b) $1.6 \times 10^{-2} \mathrm{MPa}$

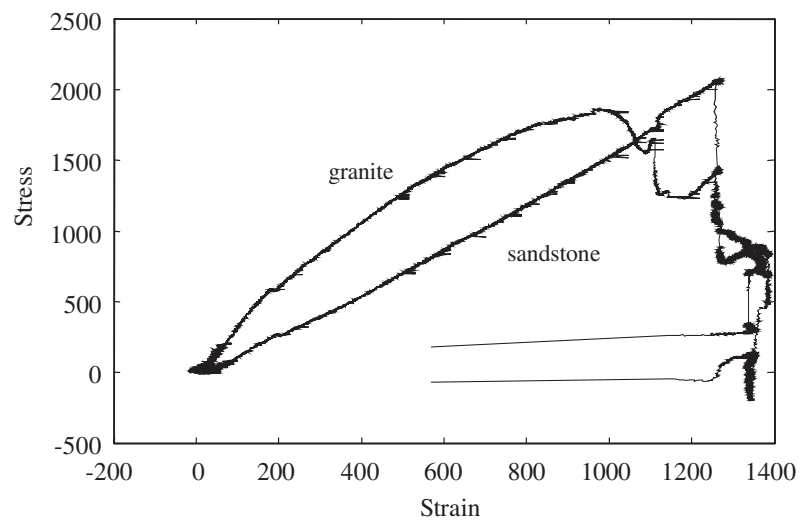

Figure 3

The stress-strain variations of rock sample during the loading process (a), and the stress-strain curve of horizontal shear (b).

still others the strain of which remained basically unchanged. XU et al. (1989; 2001a,b) and other authors (Mogi, 1984; Hu et al., 1997) have pointed out these characteristics of short-term and imminent strain precursors frequently. These characteristics appeared again in the present study; this has formed an important and feasible experimental basis for us to predict the time of earthquake occurrence.

\subsection{Characteristics of the Variation of AE Frequency and Intensity with Time During} the Loading Process of Rock Sample

The statistical averages of the number of $\mathrm{AE}$ events per $10 \mathrm{~s}$ were determined, with which the variation of $\mathrm{AE}$ rate with time during the loading process of rock 


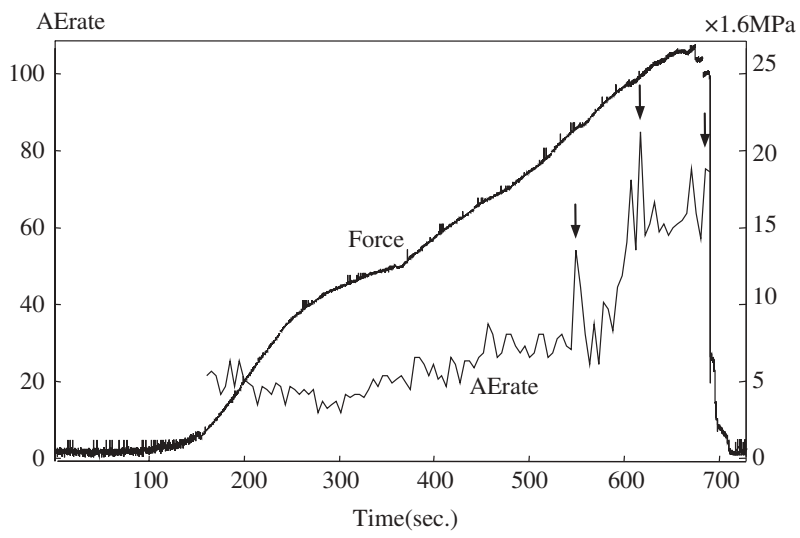

Figure 4

The variation of AE frequency with time during the loading process of rock sample.

samples was plotted, as shown in Figure 4. Arrows in the figure indicate the time position of occurrence of each large fracture. It can be seen from the figure that the number of microcracking events (AE rate) fluctuated more frequently (largely) when the extra large fracture was impending. There are some relatively longer time periods of AE active and quiet alternation; among them, three are most remarkable. Before each extra large fracture, the AE rate always increased (clustered) first, became relatively quiet next, then reactivated (clustered), and finally the extra large fracture occurred.

The intensity of extra event of AE is considerably higher than that accompanying other events. When viewed from the waveform record (as shown in the following), they were all amplitude-limited and lasted a very long time. In this paper, the size (intensity) of an event is determined according to the length of its time duration. The formula is

$$
M=c+d \log \left(T_{c}-T_{0}\right)
$$

where $T_{0}$ is the time of fracture occurrence; $T_{\mathrm{c}}$ is the ending time of the coda, i.e., the corresponding time when the amplitude becomes smaller than or equal to a certain specified value. In case that the record was amplitude-limited or incomplete, $T_{\mathrm{c}}$ can be determined by the formula given below. Assuming that the wave train amplitude of events obeys the rule

$$
A=A_{0} t^{-k}
$$

$T_{\mathrm{c}}$ can be determined once $A_{\mathrm{c}}$ is given.

Figure 5 shows the variation of AE intensity with time before and after the first extra large fracture during the loading process of rock sample. It is seen from the figure that in a period of time before the extra large fracture (the highest vertical line 

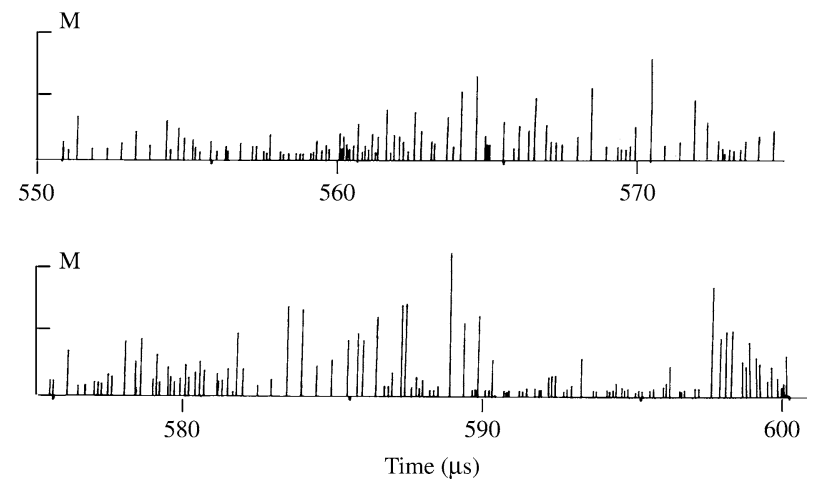

Figure 5

The magnitude variation of $\mathrm{AE}$ events with time before and after the first extra large fracture during the loading process of rock sample.

in the lower part of the figure), AE events were of a large number but low intensity; later on, they became less in number but were of higher intensity; finally, the extra large fracture occurred when the frequency of AE events increased again. Before the preceding large fracture, AE events also showed characteristics similar to those mentioned above. If there are very few large AE events but a few good small ones to occur after the large fracture, there might be another large fracture to follow; otherwise, no large fracture would reoccur. The status for the second and third extra fractures is similar to this, and hence the figures are omitted here.

\subsection{Characteristics of the Variation of Microcracking Waveform During the Loading Process of Rock Sample}

Before and after the three extra large fractures of rock sample, the waveforms of a few fractures are of basically similar characteristics. In the early stage, most of the fractures were of an isolated type. With the increase of stress and the impending extra large fracture, the types of fractures tended to be complete. There were various types of fractures to occur, such as the types of fore-main-after-fracture fore-mainfracture, main-after-fracture, and swarmed fracture. Figure $6 \mathrm{a}$ is the waveform record of a typical fore-main-after-fracture type. Other types can be regarded as part of it. In the swarmed-fracture type, the amplitudes (sizes) of events are close to one another. This type is thoroughly discussed elsewhere. Moreover, with the pending extra large fracture, there would be events that contain obviously more lowfrequency components in the waveform to occur. Figure $6 \mathrm{~b}$ is a record of this type. It is obvious that the low-frequency components in Figure $6 \mathrm{~b}$ are more than those in Figure 6a. Figure $6 \mathrm{c}$ is the first extra large fracture and Figure $6 \mathrm{~d}$ is the after-fracture nearest this extra large fracture. The waveform in Figure $6 \mathrm{c}$ is seriously amplitudelimited, showing that this event is of a much higher intensity than other events. 
(a)

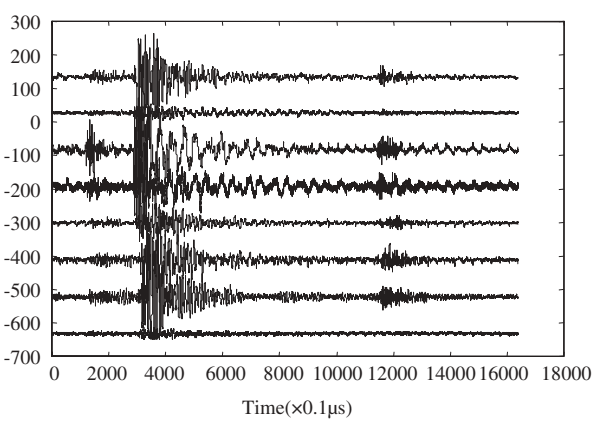

(c)

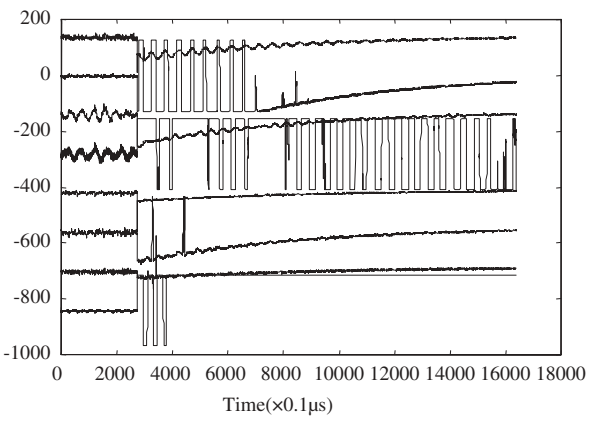

(b)

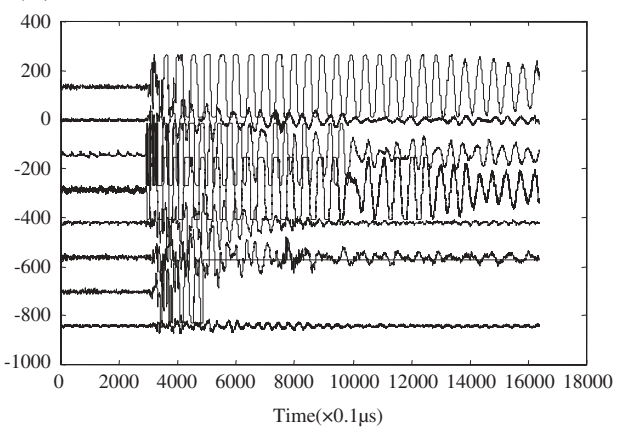

(d)

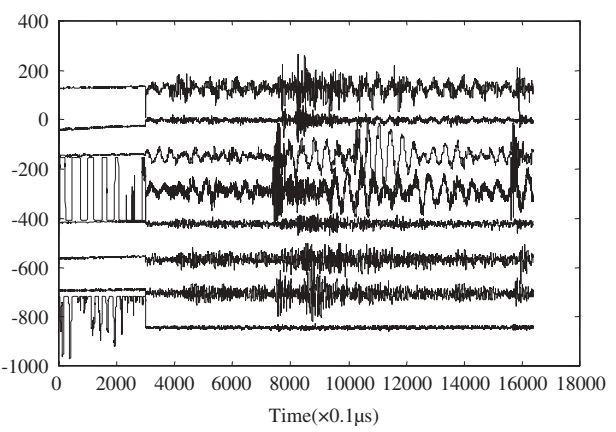

Figure 6

Waveform records of some fractures before and after the extra large fracture of rock sample (a) Highfrequency (wave) fore-fracture; (b) Low-frequency (wave) fore-fracture; (c) The first extra large fracture; (d) After-fracture nearest the extra large fracture.

Figure 6d shows that there were not only high-frequency (wave) events, but also lowfrequency (wave) events after the extra large fracture. It is noteworthy that, to the extent low-frequency (wave) events exist, there remains the possibility for large or even larger fractures to occur.

\subsection{Characteristics of the Variation of Shadow Areas of Caustics During the Fracture Preparation Process of the Plexiglass Sample}

In the experiment, the shadow areas of caustics were clear and obvious, as shown in Figure 7. At the crack tip, there is an obvious dark shadow area which has an obvious demarcation line with the high-density fringe area that neighbors it. The bright demarcation line at the crack tip is the caustic curve, and the dark shadow area is the shadow area of caustics (for the local characteristics, see Figure 8c). They grew with increasing load and reduced with decreasing load. Figure 7 shows that the shadow grew with the increase of pressure. Untill the late stage, shadows occurred 


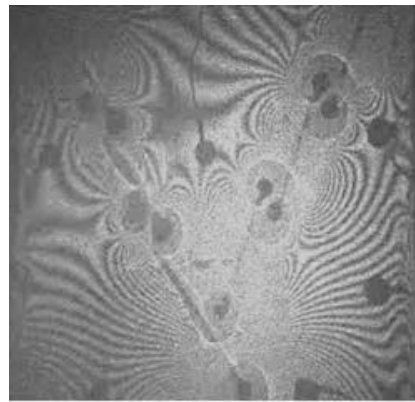

(a) $8.3 \mathrm{MPa}$

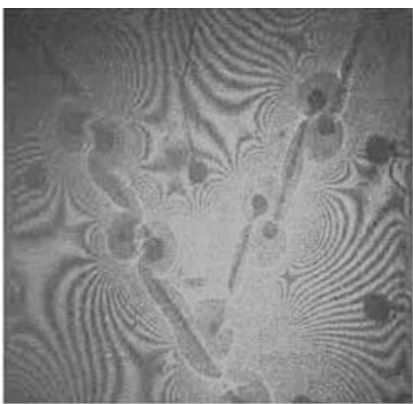

(b) $20.8 \mathrm{MPa}$

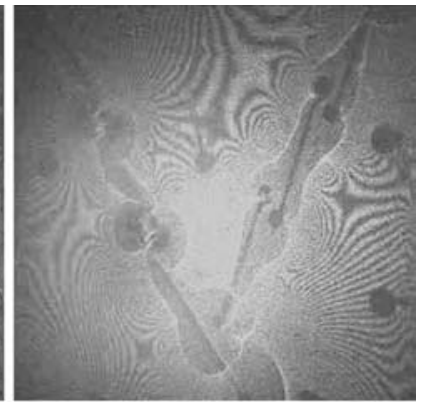

(c) $31.2 \mathrm{MPa}$

Figure 7

The variation of shadow areas of caustics with loading.

not only at the crack tip but also along the crack surface. The growth of these shadow areas means that the frictional action between crack surfaces had become fairly strong. In due time, the shadows between crack surfaces overlapped with those at the crack tip. When the shadow area of caustics grew from small to large to a certain degree, (micro) cracks occurred, which were just in the shadow area of caustics (for more details, see XU et al., 2002a,b). After this, the shadow area of caustics no longer grew and kept approximately unchanged.

\subsection{Characteristics of the Variation of Stress Intensity Factors During the Fracture} Preparation Process of the Plexiglass Sample

Experimental mechanics shows that, for cracks of pure mode II, the typical caustic curves under different conditions are as shown in Figures $8 \mathrm{a}$ and $8 \mathrm{~b}$. Figure 8a gives the negative real image of transmitting light, negative virtual image of reflecting light, and so on, while Figure $8 \mathrm{~b}$ gives the positive real image of
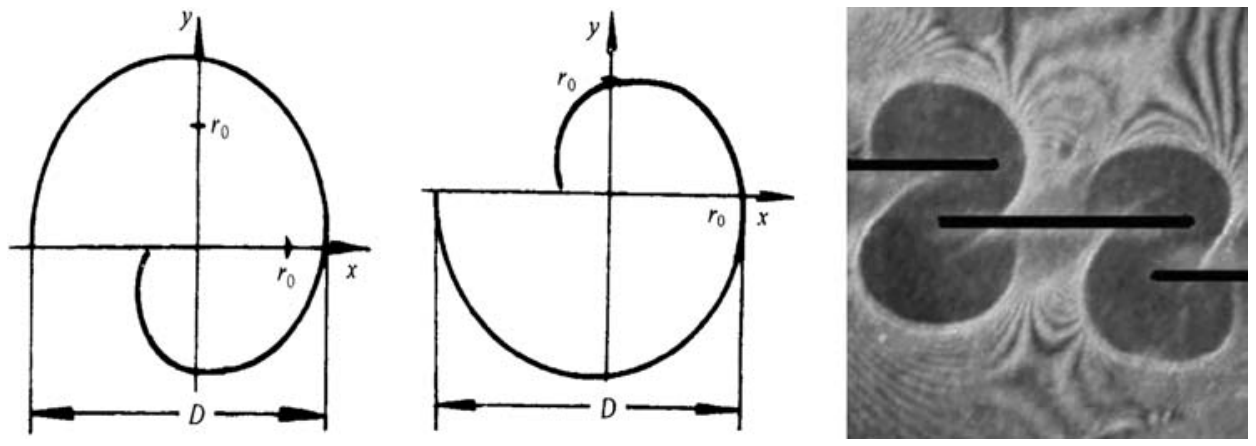

Figure 8

Schematic diagrams of typical caustic curves (a and b) and patterns recorded in this study (c). 
transmitting light, positive virtual image of reflecting light, and so on (Su and ZHIHUI, 1987; Su and ZHENG, 1997). Figure 8c gives the typical record of the present experiment.

The empirical formula for calculating SIFs by use of caustic curves is (Su et al., 1987, 1997)

$$
K_{\mathrm{II}}=2(2 \pi)^{1 / 2} D^{5 / 2} /\left[3(3.02)^{5 / 2}\left|z_{0}\right||c| d \lambda_{m}^{\frac{3}{2}}\right]
$$

where $d$ is the sample thickness; $z_{0}$ the distance from the image plane of caustics to object (sample) plane; and $D$ is called the characteristic length of caustic curve. For elastic materials, $D$ is the only variable on the right-hand side of Eq. (3) when the experimental setup is given. In the present experiment, the cracks are inclined. When a single inclined crack is under tension, the SIFs are

$$
\begin{gathered}
K_{\mathrm{I}}=\sigma(\pi a)^{1 / 2} \sin \beta \sin \beta=K_{\mathrm{Is}} \sin ^{2} \beta, \\
K_{\mathrm{II}}=\sigma(\pi a)^{1 / 2} \sin \beta \cos \beta=K_{\mathrm{IIs}} \cos \beta .
\end{gathered}
$$

This means that the calculation result of cracks of pure mode I or II differs from that of the single inclined crack in the tension state only by a constant related to the angle between the crack and loading direction. In relevant literature (YIN, 1985), the result is considered suitable for compression too. In fact, the friction between crack surfaces under compression may increase; this has also been confirmed in the present experiment.

There are a total of six macroscopic cracks in the sample. The SIFs for the tips of the six cracks are determined by Eq. (3). The six cracks gave a total of nine relatively clearer measuring points. Three cracks had two measuring points each, the remaining three had one measuring point each, hence their SIFs are average results. The tips of three cracks were close to the sample boundary, which may cause errors in the results.

The results are as shown in Figure 9. During the entire loading process, the rate of variation of the SIF was not the same. With the increase of pressure, there were mainly three stages of variation of the SIF. In the first stage, the SIFs increased very rapidly; however, the rate and time of increase were not the same because the states of macroscopic cracks were not all the same. In the second stage, the SIFs had an obvious change, and the rate of variation reduced evidently. In the third stage, the SIFs tended to be close or equal to the fracture toughness and the cracks began to propagate. The fracture toughness values obtained from the six cracks are approximately the same, except those from cracks Nos. 1 and 4 which are slightly on the high side. At that time, the SIF at the crack tip had already reached the fracture toughness and hence the external load mainly played the role of supplying the energy for fracture propagation. When the energy accumulated to a certain degree, there would be microcrack to occur so that the energy could be released. For 

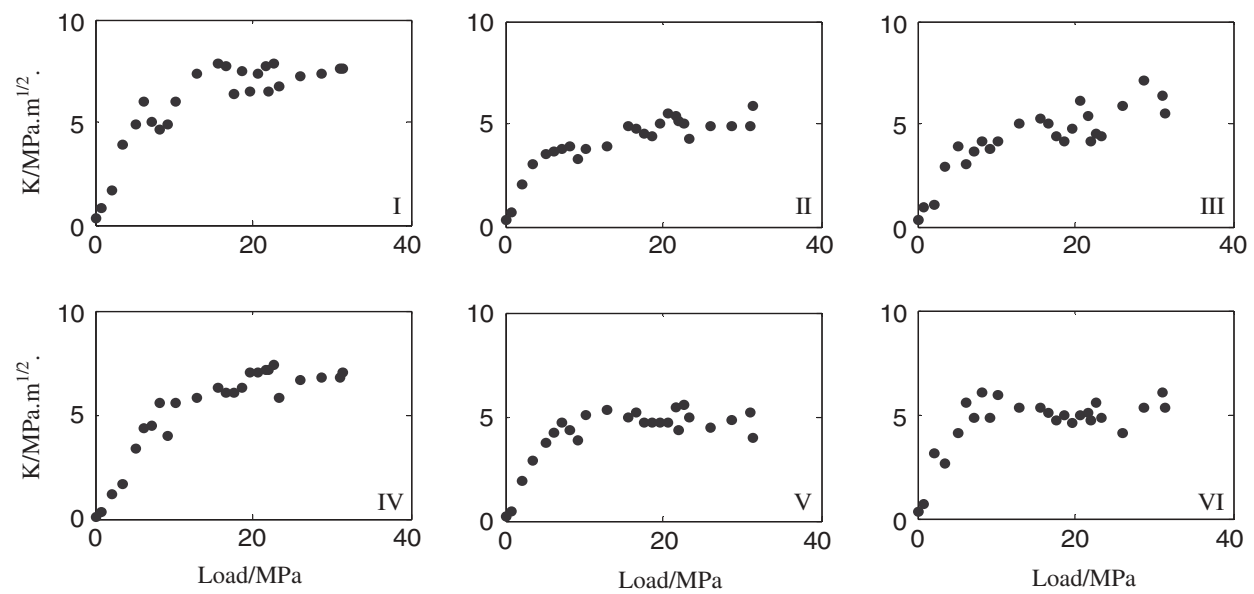

Figure 9

The variation of SIFs of the six cracks with time.

the production of the next fracture, a new turn of energy accumulation is required. Such a process manifests itself on the SIF diagram as a fluctuation around the fracture toughness.

\subsection{Characteristics of the Variation of Microcracking Waveforms During the Fracture} Preparation Process of the Plexiglass Sample

Like that in the rock sample, most of the microcracking events during the fracture preparation process were high-frequency (wave) events; the variation of their frequency and intensity was also similar to that in the rock sample. However, before an extra large event or a large event, often low-frequency (wave) events would occur, and they were considerably nearer the extra large fracture. Typical records are as shown in Figure 10. In the figure, the extra large fracture was seriously amplitudelimited, but the fore-fracture just before it was a low-frequency (wave) event; the frequency of that event was much lower than that of fractures before.

\section{Discussion and Conclusions}

\subsection{On the Earthquake Recurrence Period}

There are two kinds of concepts pertaining to about the earthquake recurrence period: One is the time separation for earthquakes to recur at the same place; the other is the interval for the active-quiet alternation of earthquakes in a larger region. However, the implication obtained from the experiment is that, regardless of which kind the recurrence period is, the most basic condition is that enough energy must be 


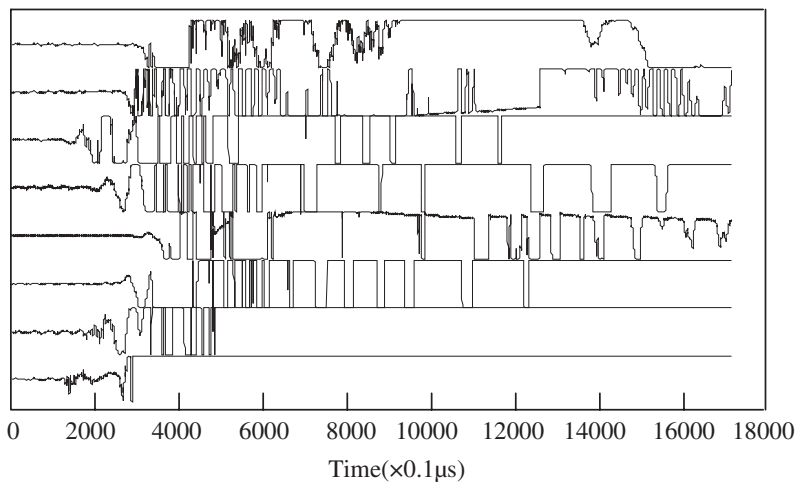

Figure 10

Records showing that low-frequency (wave) events were much nearer the extra large fracture.

accumulated in the relevant region; an earthquake ought to occur once the condition of instability failure has been satisfied. The duration in which the energy stays at a sufficiently high level is the seismic active period; in contrast, the duration in which the energy stays at an level insufficiently high is the seismic quiet period.

Fracture mechanics takes the fracture toughness $K_{\mathrm{c}}$ as a parameter of materials; it represents a critical value the SIF should have when the crack begins to propagate unstably. Correspondingly, the crack growth resistance $G_{\mathrm{c}}$ has also been specified. It represents the energy needed for creating a unit area of new crack surface or a critical value the energy release rate should have when the crack begins to propagate unstably. For (regular) cracks of pure modes I, II and III, these two quantities have a constant relation with each other (YIN, 1985). The energy release rate is

$$
G=\partial U / \partial A \text {. }
$$

It represents the strain energy released by the unit area of new surface created by crack propagation.

$$
G=G_{\mathrm{I}}+G_{\mathrm{II}}+G_{\mathrm{III}}=\frac{K_{\mathrm{I}}^{2}}{E^{\prime}}+\frac{K_{\mathrm{II}}^{2}}{E^{\prime}}+(1+v) \frac{K_{\mathrm{III}}^{2}}{E},
$$

where

$$
E^{\prime}=E
$$

for plane stress, and

$$
E^{\prime}=E / 1-v^{2}
$$

for plane strain. 
The criterion for crack propagation is that the crack propagates unstably when $K \geq K_{\mathrm{c}}$ or $G \geq G_{\mathrm{c}}$. For cracks of mixed mode, the theory of strain energy density factor or maximum energy release rate is usually used as the criterion for crack propagation. The strain energy density $U$ and strain energy density factor $S$ are, respectively

$$
U=(1 / 2) \sigma \varepsilon=S / r
$$

and

$$
S=a_{11} K_{\mathrm{I}}^{2}+2 a_{12} K_{\mathrm{I}} K_{\mathrm{II}}+a_{33} K_{\mathrm{III}}^{2}
$$

The theory of strain energy density assumes that the crack propagates in the direction of the minimum $S$ value, $S_{\min }$; the crack begins to propagate when $S_{\min } \geq$ $S_{\mathrm{c}}$. The theory of maximum strain energy release rate assumes that the crack propagates in the direction of the maximum strain energy release rate; the crack begins to propagate when $G \geq G_{\mathrm{c}}$. In fact, the two criteria are consistent with each other.

When the strain energy or SIF in a region is high enough to satisfy the condition of unstable fracture, an earthquake occurs. The duration of this sustaining high value is the seismic active period. After earthquake, the strain energy becomes less and the SIF lowers. The time length needed for the SIF to increase again to a level that satisfies the condition of unstable fracture depends on a number of factors. One is the amplitude of reduction of the strain energy or SIF; another is the rate of stress increase; and the other is the changed value of the fracture toughness or crack growth resistance of materials. This period of time before the condition for unstable fracture has been reached is the seismic quiet period. Because the strain energy, stress rate and fracture toughness are not all the same, the seismically active and quiet periods are also different. Figure 3 shows that, when the strain energy has reached a certain value, the fracture active period comes and extra large fracture occurs. The figure also shows that, in the three periods, the strain energy density factors or critical values of energy release rate for crack propagation are not the same. This means that the material experiences change in property after the occurrence of fracture; here, this change is that the strain energy density factors or critical values of energy release rate for crack propagation have decreased. Similarly, Figure 9 also indicates that SIF is not a constant, so long as the SIF is not smaller than the fracture toughness, fractures on the sample always remain in the active period. In this way, the periodical activity of earthquakes has been explained in terms of fracture mechanics. However according to results of those experiments, even if the sample is homogeneous material, the earthquake recurrence period is not a constant, which is relative to the stress situation, property variation of material and so on.

More than two decades before, many experts began to study the explanation of earthquake activity in terms of fracture mechanics. However, the studies they made 
were mostly from the theoretical angle. Certain good methods they proposed could hardly be used; for example, the method of predicting earthquakes based on the fracture energy characteristics was not feasible then. With the development of science and technology, especially that of GPS, GIS and RS, it has enabled the precise measurement of the crustal deformation and calculation of the strain energy. This has also reopened a field of study for the prediction of earthquake activity by use of the strain energy.

\subsection{On the Trend of Post-seismic Development}

Regarding the trend of post-seismic development, there are two problems. One is whether or not there there would be even larger earthquakes to occur? The other is how large the aftershocks could be? It can be seen from the test results that if a large event is followed by events that contain more low-frequency components of a strong event in the spectrum, there might be an even larger fracture to come; in contrast, if there are more high-frequency components of a strong event, no larger events would occur. Figure 6b shows the low-frequency (wave) events a slightly longer time before the extra large event, while Figure 10 shows the low-frequency (wave) events a very short time before the extra large event. is like Figure 6b, In other words, when the extra large event is followed by low-frequency events (as Figs. 6b and 6d), there would be events with a fairly strong event to come. Furthermore, when there are too many events of low intensity but less or very few of high intensity, there would probably be events of higher intensity (in a relative sense) to come. In the upper part of Figure 5, the magnitude of events after the largest event decayed more uniformly; in contrast, in the lower part, the magnitude of events after the extra large event decayed none-uniformly, with large events being very few and small ones more, hence there were high-magnitude events to reoccur. Figure 4 shows that after the first extra event the AE rate decreased, but with the stress increasing the rate increased again, when it reached the maximum value, the second extra fracture occurred. In addition to the above, judgment must also be made relative to the state of stress and strain. After a large event has occurred, there may be large or even larger events to come when the stress (or strain energy) does not have obvious reduction or it even increases again very soon; in contrast, when the stress (or strain energy) reduces obviously or decreases considerably, there unlikely would be large or even larger events to occur later, as Figure 3 showed, the shear stress and strain still increased after an extra event, another extra fracture would occur again. Of course, the above comparison is related to the properties of materials; for example, when $K \geq K_{\mathrm{c}}$ or $G \geq G_{\mathrm{c}}$, the crack propagates unstably (earthquake occurs); otherwise, the crack does not propagate unstably (no earthquake occurs). Thus in the study of earthquake series, it is important to study the stress situation and the fracture mechanism of event. 
The problems of how to determine the crustal stress in the field and how to determine precisely the strain energy need to be studied thoroughly. Though there are still many difficulties, this has provided us with a definite and feasible direction towards which efforts should be made.

\section{Acknowledgement}

This project is supported by the NSFC (10232050), MOST(2004CB418406) and Key Projects of 10th Five-Year Plan of Yunnan Province, documented as yunnandiqu [2002] 540202.

\section{REFERENCES}

CAI JINGGUAN, and LiU ZHENGRONG (1990), Forthcoming strong earthquakes calculated upon recent seismic activities, Earthquake Research in China 3, 47-52 (in Chinese with English abstract).

Hu Yili, Xu Zhaoyong, Wang Bin, Zhao Jinming, and Mei Shirong, (1997), The variation of strain field on free plane of the sample containing hard inclusion in process of fracture development, Earthquake 2, 162-168 (in Chinese with English abstract).

Mei Shirong, (1960), The seismic activity of China, Acta Geophysica Sinica, 1, 1-19 (in Chinese with Russian abstract).

MoGI, K. (1962), Study of the elastic shocks caused by the fracture of heterogeneous materials and its relation to earthquake phenomena, Bull. Earthq. Res. Inst., Univ. Tokyo 40, 125-173.

Mogi, K. (1963), Some discussions on aftershocks, foreshocks and earthquakes swarm - The fracture of a semi-infinite body caused by inner stress origin and its relation to the earthquake phenomena (3) Bull. Earthquake Res. Inst. Univ. Tokyo 41, 615-658.

Mogi, K., Fundamental studies on earthquake prediction, A Collection of Papers of International Symposium on Continental Seismicity and Earthquake Prediction (ISCSEP) (ed.The Organizing Committee of ISCSEP), (Beijing: Seismological Press,1984), pp. 619-652.

Shi Zhenliang, Huan Wenlin, Lu Shoude, and Yan Jiaquan, Characteristics of continental seismicity in Central and Eastern Asia (Abstract), A Collection of Papers of International Symposium on Continental Seismicity and Earthquake Prediction (ISCSEP) (ed. The Organizing Committee of ISCSEP ) (Beijing: Seismological Press,1984), pp. 155.

Su Xianis, and Lei Zhinui, (1987), A preliminary application of the experimental method of dynamic caustics in fracture mechanics, Acta Mechanica Sinica 19, 357-365 (in Chinese with English abstract).

Su XIANJi, and Li Zheng, (1997), Dynamic Measurement Techniques in Solid Mechanics (Beijing: Higher Education Press, 1997) (in Chinese).

Xiong Bingheng, Wang Zhengrong, Lü Xiaoxu, Zhong Liyun, Zhang Yong’an et al. (2002), A noval optical testing system created for experimental seismological research, Chinese J. Lasers 29, 376-380 (in Chinese with English abstract).

XU SHAoxie, (1984), A review of the seismicity patterns. In A Collection of Papers of International Symposium on Continental Seismicity and Earthquake Prediction (ISCSEP) (ed. The Organizing Committee of ISCSEP) (Beijing, Seismological Press, 1984), pp. 11-42.

Xu, Z., YANG, R., ZHAO, J. et al. (2002a), Experimental study of the process zone, nucleation zone and plastic area of earthquakes by the shadow optical method of caustics, Pure Appl. Geophys. 159, 19511966.

Xu Zhaoyong, Mei Shirong, Yang Runhai et al. (2001a), Study on short-impending strain precursors before rock sample failure, J. Seismol. Res. 24, 191-196 (in Chinese with English abstract). 
Xu Zhaoyong, Shu Yanhua, and Bao Yifeng, (1989), The rupture characteristics of rock samples with cut crack filled by epoxy resin under uniaxial compression. In A Collection of Research Papers of Experimental Geophysics, (ed. Editorial Committee of Chinese Journal of Geophysics) (Beijing, Seismological Press,1989) pp. 69-274 (in Chinese with English abstract).

Xu Zhaoyong, Yang Runhai, Wang Bin et al. (1998) Burst strain disturbance - A new test study on fracture precursor, Acta Seismologica Sinica 11, 737-744.

Xu Zhaoyong, Mei Shirong, Yang Runhai et al. (2001b), Experiment study of the precursor in far and ultra-far field, J. Seismol. Res. 24, 332- 338 (in Chinese with English abstract).

XU ZhaOyong, YANG RunhaI, ZhaO JinMing et al. (2002b), Experimental study of multiple fractures on a $Y$-shape boundary of blocks, Chinese J. Geophy. 45(Sup.), 214-224 (in Chinese with English abstract).

Yin Xiangchu, Solid Mechanics (Beijing: Seismological Press, 1985) (in Chinese).

Zhang Guomin (1987), Rhythmic characteristics of high seismic activity in China mainland, Seismol. Geol. 9, 27-37 (in Chinese with English abstract).

Zhang Guomin, Geng Luming, and Shi Yaolin, (1993), A computer model for cyclic activities of strong earthquake in continental seismic zones, Earthq. Res. in China 9, 20-32 (in Chinese with English abstract).

(Received October 26, 2004, revised/accepted September 26, 2005)

\section{To access this journal online: (28) http://www.birkhauser.ch}

